\title{
Respiratory rehabilitation with abdominal weights: a prospective case study
}

\author{
Stanley John Winser ${ }^{1}$, Priya Stanley ${ }^{1}$, George Tarion ${ }^{2 *}$ \\ ${ }^{1}$ Department of Physiotherapy Masterskill University college of Health Science (MUCH), Cheras, Malaysia; \\ ${ }^{2}$ Department of Physical Medicine and Rehabilitation, Christian Medical College, Vellore, India; *Corresponding Author: \\ stanjwpt@gmail.com, stanjw_pt@,yahoo.com, Stanley@masterskill.edu.my
}

Received 6 January 2010; revised 21 January 2010; accepted 24 January 2010.

\begin{abstract}
Objective: Abdominal weights was used to strengthen the diaphragm of a C6 ASIA (A) tetraplegic subject with the aim of studying the long term effect of the technique as a part of respiratory rehabilitation. Setting: Department of Physical Medicine and Rehabilitation, Christian Medical College, Vellore, Tamil Nadu, India. Study Design: Prospective case study. Material and methods: The peak EMG amplitude of the diaphragm (DIA), intercostals (INT) and sternoclidomastiod (SCM) were assessed using a surface EMG and inspired lung volume (ILV) was assessed using an adjustable portable spirometer. The measurements were repeated after 3, 6, 9 and 12 months of inspiratory muscle training for a period of 15 minutes daily, 6 days a week for 12 months. Results and discussion: Peak amplitudes recorded by the EMG of DIA and SCM muscles showed a progressive increase, INT muscle did not show a consistent change. INV showed a gradual rise from $1772 \mathrm{ml}$ to $2760 \mathrm{ml}$ over the study period. These values have the following significance: 1) Use of abdominal weights as a part of respiratory rehabilitation has beneficial long term effects; 2) In patients with tetraplegia, respiratory muscles in particular the diaphragm, are trainable in terms of muscle efficiency; 3 ) The improvement in the muscle efficiency obtained during the early rehabilitation can be maintained or improved using simple non sophisticated exercises like abdominal weights post discharge. Conclusions: Abdominal weights can be used as an effective adjunct to pulmonary rehabilitation in improving the efficiency of diaphragm on a long term basis, thereby reducing the risks associated with pulmonary complications.
\end{abstract}

Keywords: Tetraplegia; Abdominal Weights; EMG

\section{INTRODUCTION}

Complete lesions of the spinal cord affect the respiratory inspiratory and expiratory muscles. The degree of impairment in respiratory function is related to the level of the lesion [1]. If the lesion is below the C3-C4 level, the diaphragm is intact but the loss of other respiratory muscles decreases the vital capacity (VC) to approximately $50 \%[2,3]$ and total lung capacity (TLC) to approximately $70 \%$ [3-5] of predicted normal values. Paralysis of the expiratory muscles reduces the ability to force expiration leading to an increased residual volume and reduced ability to huff (forcefully exhale) and cough. This may cause secretion to accumulate in the airways. In complete lesions above the Th6 level, the autonomic nervous system is injured, and bronchial hypersecretions occur, which further aggravates problems regarding secretions [6].

Currently a complete retrival of the muscle efficiency following spinal cord injury (SCI) is not known. Cases in which patients are unable to maintain adequate ventilation, long-term mechanical ventilator support is indicated [7]. Although this treatment is effective, it can also lead to serious medical complications such as infection, pneumonia, atelectasis, and even death [8-10]. In fact, the primary cause of death after SCI, regardless of the level of injury, is caused by respiratory insufficiency and complications associated with impaired respiratory function.

Training of these muscles may improve the daily activities. Respiratory muscle training in healthy subjects, COPD (Chronic Obstructive Pulmonary Disease) and other pulmonary patients has shown positive effects [11-17]. In individuals with tetraplegia, training of the inspiratory muscles has been applied by Gross et al. [18] Biering-Sorensen et al. [19] and Derrickson et al. [20]. Use of abdominal weights, resistive inspiratory muscle training, Abdominal binders [21], Trendlenbergs position and Incentive spirometry [22] have been reported in literature to have useful effects in improving the effi- 
ciency of diaphragm among the target population. In the survey done we found a paucity of literature on the long term effects of these therapeutic modalites on the respiratory status of tetraplegic patients, thus we intended to observe the influence of abdominal weights on the efficiency of the available respiratory muscle on a tetraplegic patient over a period of 1 year.

\section{CASE REPORT}

A 30-yr-old male patient presented with complaints of weakness of arms and legs, with fecal and urinary incontinence, in March 2004. The patient's history revealed that a $\mathrm{C} 5$ vertebrate fracture caused SCI after a fall from height (mango tree) in December 2003. He had applied to a clinic and had been managed conservatively with bed rest for 3 months.

The patient's history included an internal fixator at his femur because of a fracture that occurred in another vehicle accident before 6 yrs. The patient's medical status was stable. He had no pressure ulcers, range of motion (ROM) of all four limbs were full and free. On clinical neurologic examination, he had spastic tetraplegia with loss of motor function below the C6 level and loss of all sensation below C7 dermatome. Spasticity was found to have grade $1+$ in Modified Asworth scale (MAS) in all 4 limbs. Deep tendon reflexes were hyperactive, and plantar response was extensor bilaterally. The patient was classified as C6 American Spinal Injury Association grade $\mathrm{A}$ and admitted to a rehabilitation program. The rehabilitation program involves concurrent sessions of physiotherapy, occupational therapy, vocational training, prosthetics and orthostics and recreation classes. In the department of physiotherapy the patient was undergoing re-education exercises for the upperlimbs, progressive orientation to erect stance using tilt table to counteract postural hypotension, passive movement for the lowerlimbs, passive stretching for hamstrings and tendo achilles. In addition to this the patient was given strengthening to the diaphragm muscle using Abdominal weights as a part of respiratory rehabilitation.

\section{RESPIRATORY ASSESSMENT}

Patient had no history of COPD, TB or other chronic respiratory illnesses. Patient demonstrated a diaphragmatic breathing pattern. Chest expansion was $2 \mathrm{cms}$ at the level of xiphisternum and nil at nipple and axilla. Cough was weak functional. Auscultatory findings revealed a good air entry in all lobes and no added sounds were recorded. Muscle strength of the Diaphragm was good. The strength of the diaphragm involves interpretation of the total neurological involvement, complete respiratory evaluation and observational techniques. It is best done in supine lying. "Poor power" is being graded if the subject is not able to expand his/her epigastric region fully on deep inspiration. "Fair power", if the subject is able to expand his/her epigastric region fully on deep inspiration. "Good power" the therapist's hands are placed over the epigastric region with fingers spread, and the subject is asked to inhale, while maximum manual resistance is applied. If the subject is able to complete a full epigastric raise against resistance then helshe can be graded as Good. The subjects who are able to take resistance but not able to hold can be graded as "Fair plus" [23]. Subjects with diaphragmatic power of fair plus and above are candidates for progressive resisted exercises.

\section{MATERIALS AND METHODS}

A prospective case study of a 30 year-old tetraplegic patient, with complete spinal cord section at C6 level, was considered. The subject gave written consent to participate in the trial, the study was approved by the research board of Christian Medical College, Vellore, India. Initial assessment was done in march 2004 and subsequent assessments were planned at an interval of three months for the next one year. The 2 outcome measures which were considered are peak amplitude of Electromyographic (EMG) readings of diaphragm, intercostals and sternocliedomastoid muscles and Inspired Lung Volume (ILV). The investigator who performed these tests was blind to the situation.

\subsection{EMG Analysis}

The EMG activity was measured using 3 pairs of Silver chloride bipolar surface electrode. The active electrodes were placed over T7 \& T8 intercostal space, T4 \& T5 intercostal space and mid portion of sternocleidomastoid to get the electrical activity of diaphragm (DIA), intercostals (INT) and sternocleidomastoid (SCM) muscles respectively as shown in Figure 1. Readings of all three groups of muscles were taken simultaneously [24]. Electrodes were secured to the skin using adhesive plaster after skin preparation. While taking the readings the subject was instructed to take 3 consecutive deep inspirations followed by expiration, readings were recorded for 10 seconds and the mean of peak amplititudes in the EMG were recorded. Three trials were done and the best response of peak amplitude was noted.

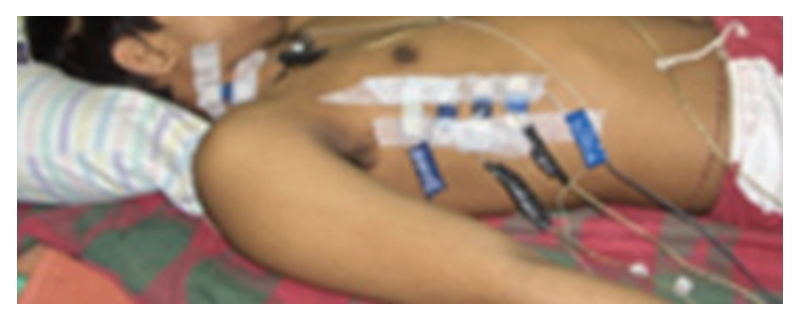

Figure 1. Placement of surface electrodes. 


\subsection{ILV Analysis}

The ILV was assessed using a volume adjustable portable spirometer (Spectra Spirometer ${ }^{\mathrm{TM}}$ ) The spirometer has calibrations for adjusting the flow rate $(100 \mathrm{cc}, 200$ $\mathrm{cc}, \ldots 800 \mathrm{cc}$ ) The device has a single chamber with 1 ball inside. Air flows into this channel on inspiration and raises the ball depending on the flow inhaled per second. This upward movement of the ball during inspiration provides the patient with visual feedback i.e. an indirect indicator of the inspired volume. The patient was instructed to perform a deep inspiration through the mouth piece and while inspiring he was required to keep the ball inflated for the maximum seconds possible. As shown in Figure 2.

ILV was measured using the formula: ILV = Flowrate $\times$ seconds

Flow rate: was kept constant at $400 \mathrm{cc}$. Seconds: was a measure of patients ability to keep the spirometer ball inflated during inspiration and was calculated using a stop watch.

\subsection{Treatment Protocol}

Diaphragm was strengthened using abdominal weights for 15 minutes/day for six days/week for a period of 1 year. An initial evaluation of weight to be placed over the abdomen was done. The subject was positioned in supine lying, then a minimal weight was placed over the epigastric region (weights starts with half a kilogram) and then the subject was allowed to breath (the weight should come up fully with each inspiration). If the subject showed any signs of fatigue or started using his accessory muscles, the weights were taken off immediately. Adequate rest was given, then the procedure was repeated using a lesser weight. If the patient is able to take up the weight comfortably for 15 minutes, a short break was given and the procedure was repeated by increasing the weight by half a kilogram, thus the appropriate weight for training the diaphragm was determined by trial and error method [23]. The evaluated weight was placed over the epigastric region with an isosceles triangular board. The board was placed in such a way that one of the corners touches the xiphisternum and the other two corners touching the anterior borders of the ribcage. Cushioning is provided with the under surface of the triangular board in order to prevent skin irritation, as shown in Figure 3. Evaluated weights were added to the triangular board using dish weights. During the training period the subject was instructed to perform the exercise in supine lying and do normal breathing with the weights on for 15 minutes. Progression in weights was done during each review. Subsequent assessments were planned during the routine medical screening and checkups of the client.

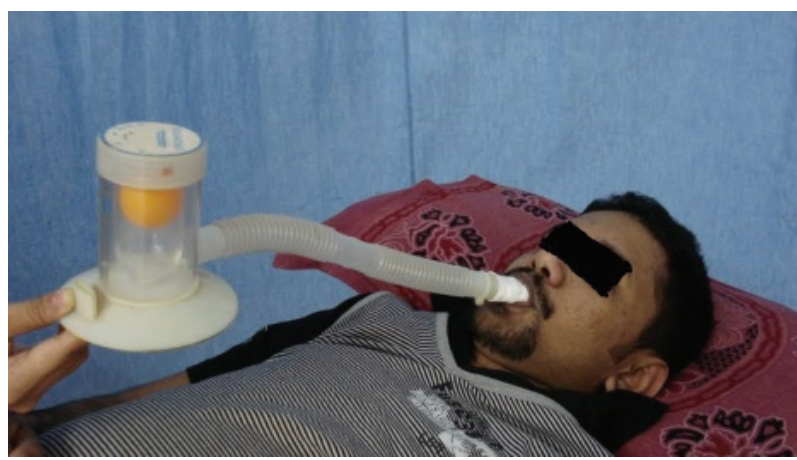

Figure 2. Analysis of ILV using spirometer.

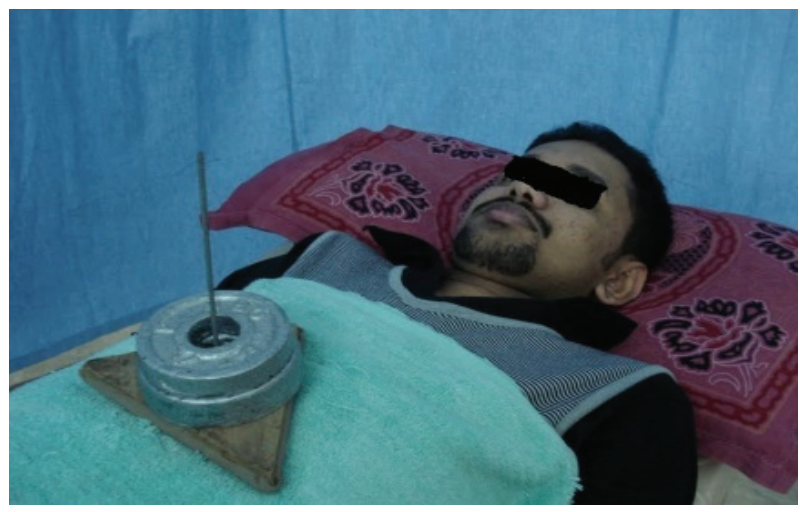

Figure 3. Placement of abdominal weights.

\section{RESULTS}

Weights were progressively increased during each visit. Initial weight was $5 \mathrm{kgs}$ and the patient progressed to 12 $\mathrm{kgs}$ by the end of the trial, as shown in Table 1.

Increment in the mean of peak amplitudes of EMG values were noted in DIA and SCM muscles, progress in the INT muscle did not show a consistent change (shown in Figure 4). Similarly the INV showed a gradual rise from $1772 \mathrm{ml}$ to $2760 \mathrm{ml}$ over the study period (shown in Figure 5).

\section{DISCUSSION}

The efficiency of respiratory muscles were studied over a period of 1 year, following administration of abdominal weighted training with the aim to determine the long term effects of the examined respiratory muscle training. The subjects respiratory muscle efficiency was assessed using recordings made over key respiratory muscles with the help of surface EMG. EMG was chosen because it captures the electrical activity happening within the muscle which is a direct measure of maximal contraction of the assessed muscle. Work done by Jennifer Beck et al on healthy individuals states there is no artifactual effect of lung volume on the diaphragm EMG signal strength during voluntary contractions [25]. Thus the EMG readings 
Table 1. Progress of the study.

\begin{tabular}{|c|c|c|c|c|}
\hline DATE & $\begin{array}{c}\text { Weights used } \\
\text { in Kgs }\end{array}$ & \multicolumn{2}{|c|}{$\begin{array}{l}\text { EMG in milli } \\
\text { volts }\end{array}$} & INV in $\mathrm{ml}$ \\
\hline \multirow{3}{*}{ Day 1} & \multirow{3}{*}{3} & DIA & 1.2453 & \\
\hline & & INT & 0.7237 & 1772 \\
\hline & & SCM & 1.5234 & \\
\hline \multirow{3}{*}{3 months } & \multirow{3}{*}{5} & DIA & 1.4356 & \\
\hline & & INT & 0.5634 & 2080 \\
\hline & & $\mathrm{SCM}$ & 1.5735 & \\
\hline \multirow{3}{*}{6 months } & \multirow{3}{*}{8} & DIA & 1.5643 & \\
\hline & & INT & 0.8675 & 2280 \\
\hline & & SCM & 1.6876 & \\
\hline \multirow{3}{*}{9 months } & \multirow{3}{*}{10} & DIA & 1.7536 & \\
\hline & & INT & 0.7457 & 2440 \\
\hline & & SCM & 1.7453 & \\
\hline \multirow{3}{*}{12 months } & \multirow{3}{*}{12} & DIA & 1.9675 & \\
\hline & & INT & 0.9656 & 2760 \\
\hline & & SCM & 1.8758 & \\
\hline
\end{tabular}

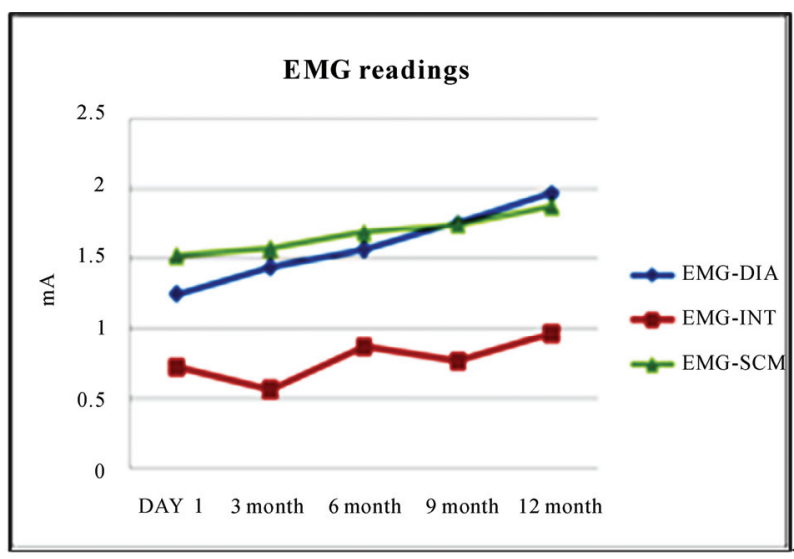

Figure 4. Peak amplitude of the analyzed muscles.

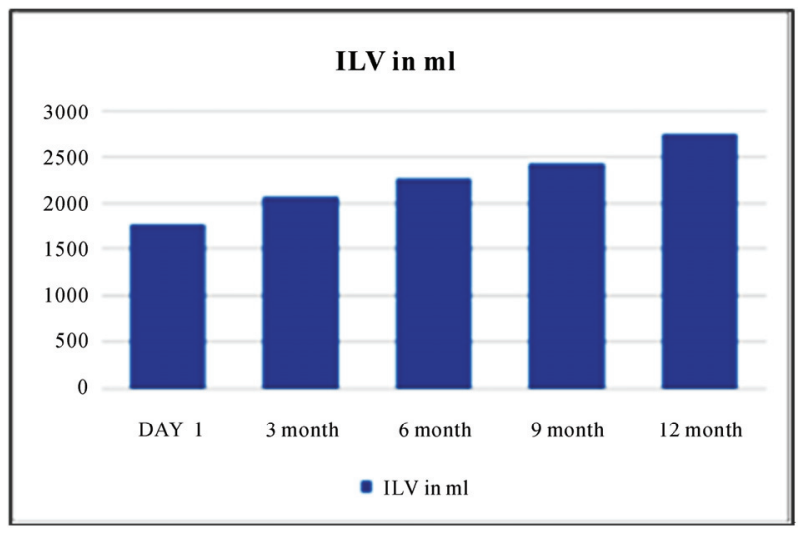

Figure 5. ILV over the study period. can be considered as an objective measure of respiratory muscle efficiency. ILV was considered as the secondary outcome measure because of the ease of administration. Though the objectivity of the test has not been tested, the results obtained are directly related to strength of the respiratory muscles and pulmonary compliance.

Our study shows the following findings:

1) Measures of EMG of the DIA and SCM muscles as well as the ILV have showed a gradual rise which implies: Use of abdominal weights as a part of respiratory rehabilitation has beneficial long term effects; 2) In patients with tetraplegia, respiratory muscles in particular, the diaphragm is trainable in terms of muscle efficiency, provided that the muscle performance is not severely compromised as in cases of ultra high lesions who require ventilator assistance; 3) The improvement in the muscle efficiency obtained during the early rehabilitation can be maintained or improved using simple non sophisticated exercises like abdominal weights. Clinically we did not observe any deterioration in the patients respiratory as well as functional status which is a good sign for a tetraplegic.

We hypothesized a reduction in the EMG activity of the sternocleidomastoid muscle following training, due to an improvement in the efficiency of diaphragm, which is the primary respiratory muscle. We found an increase in the activity of sternocleidomastoid muscle on the subject along with the increase in activity of the diaphragm. However we could not explain the reason for this phenomenon.

There were few limitations in our study which includes spasticity and adherence of the subject to the training program. There are possibilities that disturbances from the abnormally activated trunk muscles could have reduced the accuracy of EMG activity of the diaphragm and other respiratory muscles. Outcome demonstrated by the ILV has shown a consistent rise which can be considered to overcome the limitation of misleading values from the EMG if any. Though we used a log book for tracking the adherence to the treatment protocol a direct supervision of the subject on a regular basis at community level can be considered for upcoming studies, for more accurate results.

\section{CONCLUSIONS}

The peak EMG amplitude and ILV showed a consistent rise on the subject who was trained using abdominal weights. This is an apparent indication of improved performance of the target muscle with regards to strength and pulmonary function. The improvement of the muscle efficiency was maintained throughout the study period which is a positive sign of long term effects of the technique. Cost effectiveness, ease of administration of this technique ensures its frequent usage by clinical practi- 
tioners. With adequate instructions and proper training, this technique does not require direct supervision by a therapist and so is useful for community based rehabilitation. Thus we conclude stating Abdominal weights can be used as an effective adjunct to pulmonary rehabilitation in improving the efficiency of diaphragm on a long term basis, thereby reducing the risks associated with pulmonary complications.

\section{REFERENCES}

[1] Zimmer, M.B., Nantwi, K. and Goshgarian, H.G. (2007) Effect of spinal cord injury on the respiratory system: Basic research and current clinical treatment options. Journal of Spinal Cord Medicine, 30(4), 319-330.

[2] Forner, J.V. (1980) Lung volumes and mechanics of breathing in tetraplegics. Paraplegia, 18(4), 258-266.

[3] Rutchik, A., et al. (1998) Resistive inspiratory muscle training in subjects with chronic cervical spinal cord injury. Archives of Physical Medicine and Rehabilitation, 79(3), 293-297.

[4] Bake, B., Fugl-Meyer, A.R. and Grimby, G. (1972) Breathing patterns and regional ventilation distribution in tetraplegic patients and in normal subjects. Clinical Science, 42, 117-128.

[5] De Troyer, A. and Estenne, M. (1991) Review article: The expiratory muscles in tetraplegia. Paraplegia, 29(6), 359-363.

[6] Bhaskar, K.R., et al. (1991) Bronchial mucus hypersecretion in acute quadriplegia. Macromolecular yields and glycoconjugate composition. American Review of Disease, 143(3), 640-648.

[7] Jackson, A.B. and Groomes, T.E. (1994) Incidence of respiratory complications following spinal cord injury. Archives of Physical Medicine and Rehabilitation, 75(3), 270-275.

[8] Claxton, A.R., Wong, D.T., Chung, F. and Fehlings, M.G. (1998) Predictors of hospital mortality and mechanical ventilation in patients with cervical spinal cord injury. Canadian Journal of Anesthesia, 45(2), 144-149.

[9] Fishburn, M.J., Marino, R.J. and Ditunno, J.F. Jr. (1990) Atelectasis and pneumonia in acute spinal cord injury. Archives of Physical Medicine and Rehabilitation, 71(3), 197-200.

[10] Frankel, H.L., Coll, J.R., Charlifue, S.W., et al. (1998) Long term survival in spinal cord injury: A fifty year investigation. Spinal Cord, 36(4), 266-274.

[11] Akabas, S.R., Bazzy, A.R., DiMauro, S. and Haddad, G.G. (1989) Metabolic and functional adaptation of the diaphragm to training with resistive loads. Journal of Applied Physiology, 66(2), 529-535.

[12] Belman, M.J. and Shadmehr, R. (1988) Targeted resistive ventilatory muscle training in chronic obstructive pulmonary disease. Journal of Applied Physiology, 65(6), 2726-2735.

[13] Boutellier, U., BuÈ chel, R., Kundert, A. and Spengler, C. (1992) The respiratory system as an exercise limiting factor in normal trained subjects. European Journal of Applied Physiology, 65(4), 347-353.

[14] Grassino, A. (1989) Inspiratory muscle training in COPD patients. European Respiratory Journal, Supplement 7, 581s-586s.

[15] Leith, D.E. and Bradley, M. (1976) Ventilatory muscle strength and endurance training. Journal of Applied Physiology, 41(4), 508-516.

[16] Morgan, D.W., Kohrt, W.M., Bates, B.J. and Skinner, J.S. (1987) E€ects of respiratory muscle endurance training on ventilatory and endurance performance of moderately trained cyclists. International Journal of Sports Medicine, 8(2), 88-93.

[17] Noseda, A., et al. (1987) Resistive inspiratory muscle training and exercise performance in COPD patients. A comperative study with conventional breathing retraining. Bulletin European Physiopathologie Respiratoire, 23(5), 457-463.

[18] Gross, D., et al. (1980) The e€ect of training on strength and endurance of the diaphragm in quadriplegia. American Journal of Medicine, 68(1), 27-35.

[19] Biering-Sorensen, F., Lehmann Knudsen, J., Schmidt, A., Bund-gaard, A. and Christensen, I. (1991) Effect of respiratory training with a mouth-nose-mask in tetraplegics. Paraplegia, 29(2), 113-119.

[20] Derrickson, J., Ciesla, N., Simpson, N. and Imle, P.C. (1992) A comparison of two breathing exercise programs for patients with quadriplegia. Physical Therapy, 72(11), 763-769.

[21] Bodin, P., Fagevik Olsen, M. and Bake, B. (2005) Effects of abdominal binding on breathing pattern during breathing exercises in persons with tetraplegia. Spinal Cord, 43, 117-122.

[22] Kisner, C. and Colby, L.A. In: Management of pulmonary conditions. Therapeutic Exercises: Foundation and techniques, 3rd Edition, FA. Davis Company Publishers, 665-672.

[23] Wetzel, J.L. and Lunsford, B.R. (1995) In: Scot Irwin, T., Ed., Management of pulmonary conditions. Cardio pulmonary physical therapy: A guide to practice, 3rd Edition, Mosby publisher, St. Louis, 584-586.

[24] Lin, H. and Chung, C.-C. (1999) Abdominal weight and Inspiratory resistance: Their immediate effects on inspiratory muscle functions during maximal voluntary breathing in chronic tertraplegic patients. Archives of Physical Medicine and Rehabilitation, 80(7), 741-745.

[25] Jennifer, B., Christer, S., Lars, L. and Alex, G. (1998) Effects of lung volume on diaphragm EMG signal strength during voluntary contractions, Journal of Applied Physiology, 85(3), 1123-1134. 